\title{
Delayed and prolonged time for donor management including brain death determination
}

\author{
Jungsun Kim, Jisun Kwon, Seungrye Jeong, Miyoung Kim, Minyoung Chu, Jeongrim Lee, Insung Moon
}

Korea Organ Donation Agency, Seoul, Korea

Background: Delayed brain death determination or prolonged time for donor management cause increasing number of donor death, withdrawal of family's consent for donation, and increasing the expenditure for intensive care. In order to minimize the donation failure, the understanding time required for each step of organ donation is urgent.

Methods: From January 2016 to December 2018, we reviewed KODA statistical data of reported potential brain death and investigated the time intervals that take to complete each process of brain death management retrospectively. Organ donation procedures were divided into five steps. The first step started from the end of the first brain death examination to the end of the second examination, the second step was from end the second brain death examination until we got the flat electroencephalography (EEG), the third stage was from end EEG test to the declaration of brain death by brain death committee, and the fourth stage was from end of committee to entrance to operating room and the fifth step was from the operating room entrance to the completion of organ procurement.

Results: During the study period, 1,633 cases of potential brain death were managed for organ donation, and we analyzed 1,594 cases excluding donors under age of 6 . The average time spent on organ donation procedure was 33 hours and 16 minutes. The time required for each step was 11 hours 28 minutes for step 1, 5 hours 15 minutes for step 2, 5 hours 58 minutes for step 3,6 hours 38 minutes for step 4, and 3 hours 42 minutes for step 5. Among the analyzed cases, 119 cases spent more than 48 hours from step 1 to 4 , and 63 cases those included holidays in the donation process took an average of 71 hours and 5 minutes. Ninety-one cases were discontinued during the donation procedure and their causes of interruption were as follows: poor organ quality 15 cases, patient death in 15 , failure in brain death judgement in 19 , and withdrawal of donation consent in six. The time of interruption of donation procedures were as follows: 45 cases were at step 1 and 2, and the total time spent for step 1 and 2 were 16 hours and 43 minutes which accounting for half of all brain death donation procedures. In particular, the first step took average 11 hours and 28 minutes, almost twice as long as the 6-hour legal inspection interval. The third step took an average of 6 hours, which is unnecessary in foreign countries without brain death judgment committee.

Conclusions: Brain death determination procedures should be accurate without any delay. Among the measures to reduce the time, improvement of the execution of the first and second brain death examinations, prompt EEG examination, and careful approach for abolishing the brain death judgment committee are necessary. The shortage of manpower for brain death organ transplantation needs careful governmental based policy revision and social consensus.

Corresponding author: Jungsun Kim

E-mail: js.kim_a@koda1458.kr

() The Korean Society for Transplantation

This is an Open Access article distributed under the terms of the Creative Commons Attribution Non-Commercial License (http://creativecommons.org/licenses/by-nc/4.0/) which permits unrestricted non-commercial use, distribution, and reproduction in any medium, provided the original work is properly cited. 